\title{
Renal cortical necrosis at autopsy: a 12 year experience at a tertiary care centre in Mumbai
}

\author{
Fernandes Gwendolyn ${ }^{1}$, Baskaran Sumitha ${ }^{2}$, Fernandes Ellerhea ${ }^{3}$ \\ ${ }^{1}$ Dr Fernandes Gwendolyn, Associate Professor, Seth G.S. Medical College \& K.E.M. Hospital, Parel- Mumbai, \\ ${ }^{2}$ Dr Baskaran Sumitha, Final year, PG Resident, Seth G.S. Medical College \& K.E.M. Hospital, Parel- Mumbai, \\ ${ }^{3}$ Fernandes Ellerhea, Final year medical student, R.C.S.M. Govt. Medical College \& C.P.R. Hospital, Kolhapur, \\ Maharashtra, India
}

Address for Correspondence: Dr. Gwendolyn Fernandes, C-802, Swayam, Poonam Gardens, Mira Road, Thane, Maharashtra. Email: drgwenfern@yahoo.co.in

\begin{abstract}
Introduction: Renal cortical necrosis $(\mathrm{RCN})$ is an unusual cause of acute renal failure but still causes significant mortality in developing countries. Objective: To review autopsies of histologically proven RCN reported in complete autopsies over the last 12 years. Methods: All autopsies performed from 2004-2016 with RCN were analyzed retrospectively. Results: A total of 13,896 autopsies were done at this centre over this twelve year period from 2004-2016. Of these, 26 cases were diagnosed as RCN, with an incidence of $0.19 \%$. 978 of our total number of autopsies presented with Acute Kidney Injury (AKI) and the incidence of RCN in AKI was $2.7 \%$. There were 844 maternal mortality deaths during this period of which 134 (15.9\%) had AKI. RCN accounted for $2 \%$ of all maternal mortality deaths during this period. Obstetric cases amounted to $65.4 \%$ and non-obstetric cases to $34.6 \%$. Pregnancy induced hypertension; intrauterine foetal death and postpartum hemorrhage were the leading causes of RCN amongst obstetric causes while septicemia, hemolytic uremic syndrome and poisoning were the leading non-obstetric causes. Microscopically, diffuse cortical necrosis was seen in 53.8\% autopsies and patchy cortical necrosis was seen in $46.2 \%$ autopsies. Histopathology also showed intra-alveolar hemorrhage, bronchopneumonia, cerebral edema and acute respiratory distress syndrome. Conclusion: Our incidence of RCN was $0.19 \%$ at general autopsy, $2.7 \%$ in autopsies with AKI and $2 \%$ of maternal mortality deaths at a tertiary care centre.RCN in AKI being $2.7 \%$ is significantly less than the $6-7 \%$ reported in developing countries. Diffuse pattern of cortical necrosis was more common than patchy cortical necrosis.
\end{abstract}

Keywords: autopsy, acute kidney injury, anuria, diffuse cortical necrosis, oliguria, patchy cortical necrosis, pregnancy induced hypertension, renal cortical necrosis

\section{Introduction}

Renal cortical necrosis ( $\mathrm{RCN})$ is a rare cause of acute kidney injury (AKI) in developed countries, but still occurs in developing countries despite decreasing trends being reported, due to inadequate health care facilities. It carries high mortality and morbidity in developing countries accounting for 6$7 \%$ of acute renal failure, in contrast to $1.9-2 \%$ in developed countries. The incidence of $\mathrm{RCN}$ is reported $3.12 \%$ of all cases of ARF of diverse

Manuscript received: $05^{\text {th }}$ June 2017

Reviewed: $14^{\text {th }}$ June 2017

Author Corrected: $19^{\text {th }}$ June 2017

Accepted for Publication: $30^{\text {th }}$ June 2017 etiology in developing countries [1] have also been reported recently at one centre. The incidence of $\mathrm{RCN}$ reported by Kim Solez was found to be $0.2 \%$ in 11,800 autopsies in a study conducted three decades ago.

Cortical necrosis develops following ischemic necrosis of the renal cortex due to diminished renal artery perfusion secondary to vascular spasm, microvascular injury or disseminated intravascular coagulation [2]. RCN is characterized by coagulative necrosis of all the elements in the 
involved area of the cortex, including blood vessels, glomeruli and tubules. RCN is classified into complete or diffuse and patchy depending upon the extent of involvement and for prognostic purpose. Complete RCN progresses to end-stage renal disease as the damage is permanent and functional loss is irreversible, while partial renal cortical necrosis can show partial recovery of renal function. Obstetric complications are the commonest cause of RCN, and consequently the incidence of RCN is higher amongst women. The diagnosis is often suspected from the history, clinical presentation, investigations and dialysis dependency but histopathology is the gold standard for diagnosis. The present study analyses in detail renal cortical necrosis at autopsy.

\section{Aims and Objectives}

The purpose of this study was to review and analyze cases of histologically proven renal cortical necrosis (RCN) reported in complete autopsies over the last 12 years (2004-2016).

\section{Materials and Methods}

Study design: Retrospective study of all cases of all cases histologically proven renal cortical necrosis in complete autopsies done over a 12 year period, 2004-2016. Incidence of RCN in Acute kidney injury and incidence of RCN in maternal mortality autopsies were also studied.

Setting: Uropathology Division, Department of Pathology, Seth G.S. Medical College and KEM Hospital, Mumbai.

Inclusion criteria: autopsies of all ages, males and females with renal cortical necrosis diagnosed on histopathology

Exclusion criteria: all autopsies which did not have renal cortical necrosis diagnosed on histopathology.

\section{Results}

13,896 autopsies were done at this centre over the twelve year period of 2004-2016. Of these, 26 autopsies were diagnosed as RCN, with an incidence of $0.19 \% .978$ of our total number of autopsies presented with AKI and the incidence of RCN among them was $2.7 \%$. [Table 1A] There were 844 maternal mortality deaths during this period of which 134 (15.9\%) were due to Acute Kidney injury. 2\% ( 17 cases) of all maternal mortality deaths in general were due to renal cortical necrosis [Table 1B]._A wide age range from 4 days of life to 65 years was seen and there were 21 female patients $(80.8 \%)$ and 5 males patients $(19.2 \%)$. Females strongly outnumbered the males because of obstetric causes of RCN. Obstetric related cases were 17 (65.4\%) and $9(34.6 \%)$ were nonobstetric cases.
Participants: 13,896 complete autopsies were analysed.

Study size: 26 cases of renal cortical necrosis were diagnosed on histopathology from 13,896 autopsies done at this centre from 2004-2016.

Data source: Complete clinical records as well as the complete autopsy records were studied. Detailed medical history, clinical examination, clinical diagnosis, laboratory investigations, radiological reports (wherever available), complete autopsy histopathology were studied. Renal cortical necrosis was divided into 2 groups, complete or diffuse and patchy cortical necrosis.

For the histopathological examination, multiple tissue blocks of all organs were made and slides were routinely stained with Hematoxylin and Eosin. All kidney slides were stained with silver methenamine stain, Periodic acid Schiff and Fibrin stains.

Bias: Histopathogy of the 26 cases of renal cortical necrosis were reported by a single pathologist to overcome bias. RCN was divided into the following categories depending upon the involvement:

Complete or diffuse cortical necrosis: confluent global cortical destruction extending into the columns of Bertin with thin rim of subcapsular and juxtamedullary tissue is preserved. Irreversible loss of renal function is the consequence of it [1].

Patchy cortical necrosis: contiguous area of cortical necrosis up to one-third to one-half of entire cortical tissue with the potential for partial recovery of renal function [1].

All observations were recorded in Microsoft excel sheets and analysed. Data expressed as number of cases and percentages. 
Table-1A: Incidence of renal cortical necrosis $(n=26)$

\begin{tabular}{|c|c|c|c|}
\hline & $\begin{array}{c}\text { Total number of } \\
\text { cases }\end{array}$ & $\begin{array}{c}\text { Incidence of renal } \\
\text { cortical necrosis } \\
(\%)\end{array}$ & $\begin{array}{c}\text { Reference range } \\
\text { from literature } \\
(\%)\end{array}$ \\
\hline No. of general autopsies (2004-2016) & 13,896 & $0.19 \%$ & $0.2 \%$ \\
\hline No. of cases of AKI in general & 978 & $2.7 \%$ & $6-7 \%$ \\
\hline
\end{tabular}

Table-1B: Incidence of renal cortical necrosis in maternal mortality deaths $(n=844)$

\begin{tabular}{|c|c|c|}
\hline & $\begin{array}{c}\text { Total No. of } \\
\text { cases }\end{array}$ & Percentage \\
\hline No. of cases of maternal mortality deaths (2004-2016) & 844 & $100 \%$ \\
\hline No. of maternal mortality deaths with AKI & 134 & $15.9 \%$ \\
\hline No. of maternal mortality deaths with renal cortical necrosis & 17 & $2 \%$ \\
\hline
\end{tabular}

Table 2a: Etiology of renal cortical necrosis - obstetric causes $(n=17)$

\begin{tabular}{|c|c|}
\hline Etiology & No. of cases $(\mathbf{n = 1 7})$ \\
\hline Pregnancy induced hypertension \& HELLP syndrome & 7 \\
\hline Intrauterine foetal death & 7 \\
\hline Postpartum hemorrhage & 3 \\
\hline Abruptio placentae & 5 \\
\hline Sepsis & 2 \\
\hline Ruptured tubal ectopic pregnancy & 2 \\
\hline Spontaneous abortion & \\
\hline
\end{tabular}

Table 2b: Etiology of renal cortical necrosis - nonobstetric causes $(n=9)$

\begin{tabular}{|c|c|}
\hline Etiology & No. of cases (n=9) \\
\hline Sepsis & 3 \\
\hline Hemolytic uremic syndrome & 2 \\
\hline Poisoning / Toxins & 2 \\
\hline Post-operative (hysterectomy) & 1 \\
\hline Renal allograft rejection & 1 \\
\hline Perinatal asphyxia & 1 \\
\hline Acute pyelonephritis (hyperplasia - prostate) & 1 \\
\hline
\end{tabular}

Table-3: Clinical features / Clinical presentation $(n=26)$

\begin{tabular}{|c|c|}
\hline Chief complaints & $\begin{array}{c}\text { Number of cases } \\
(\mathbf{n = 2 6})\end{array}$ \\
\hline Oliguria / anuria & 21 \\
\hline Fever & 16 \\
\hline Bleeding - ecchymosis, LSCS site, subconjunctival, per vaginal, per rectal & 14 \\
\hline Hypotension & 12 \\
\hline Oedema & 6 \\
\hline Dyspnoea & 6 \\
\hline Altered sensorium/ unconscious/hepatic encephalopathy & 3 \\
\hline Diarrhoea & 3 \\
\hline Jaundice & 2 \\
\hline Pain in the abdomen & 1 \\
\hline Hematuria & 1 \\
\hline Vomiting & \\
\hline
\end{tabular}


Table-4: Laboratory parameters $(n=26)$

\begin{tabular}{|c|c|}
\hline Parameters & $\begin{array}{c}\text { Number of cases } \\
(\mathbf{n = 2 6})\end{array}$ \\
\hline Anaemia: hemoglobin- $<12 \mathrm{gm} \%$ & 19 \\
\hline Leucocytosis: total count $>12,000$ cells $/ \mathrm{mm}^{3}$ & 23 \\
\hline Thrombocytopenia: $<1.0$ lakh $/ \mathrm{mm}^{3}$ & 17 \\
\hline Raised blood urea nitrogen: $>40 \mathrm{mg} \%$ & 22 \\
\hline Raised serum creatinine: $>1.1 \mathrm{mg} / \mathrm{dl}-$ females $\& 1.3 \mathrm{mg} / \mathrm{dl}$-males & 5 \\
\hline Raised serum lactic dehydrogenase $:>300 \mathrm{IU} / \mathrm{L}$ & 14 \\
\hline Altered coagulation profile: prothrombin, APTT, fibrogen, fibrin degradation products, \\
\hline d-dimer & 3 \\
\hline
\end{tabular}

Table-5: Summary of main autopsy findings in other organs $(n=26)$

\begin{tabular}{|c|c|c|}
\hline \multicolumn{2}{|r|}{ Organ findings } & \multirow{2}{*}{$\frac{\text { Number of cases }}{5}$} \\
\hline Brain & Cerebral edema & \\
\hline & Intra-cerebral hemorrhage & 2 \\
\hline \multirow[t]{7}{*}{ Lungs } & Intra-pulmonary hemorrhage & 7 \\
\hline & Bronchopneumonia & 6 \\
\hline & Acute respiratory distress syndrome & 4 \\
\hline & Pulmonary edema & 3 \\
\hline & Interstitial pneumonitis & 1 \\
\hline & Hemorrhagic infarction & 1 \\
\hline & Collapse & 1 \\
\hline \multirow[t]{4}{*}{ Heart } & Intra-cardiac thrombi & 1 \\
\hline & Myocardial hypertrophy & 1 \\
\hline & Myocardial edema & 1 \\
\hline & Epicardial petechiae & 1 \\
\hline \multirow[t]{4}{*}{ Liver } & Centrilobular necrosis / Submassive necrosis & 4 \\
\hline & Acute fatty liver & 1 \\
\hline & Septic infarction & 1 \\
\hline & Fungal infection-Aspergillosis & 1 \\
\hline \multirow[t]{2}{*}{ Spleen } & Congestive splenomegaly & 13 \\
\hline & Septic infarction & 1 \\
\hline Pancreas & Acute necrotizing pancreatitis & 1 \\
\hline \multirow[t]{2}{*}{ Intestines } & Ischemic bowel disease & 1 \\
\hline & Fungal infection-Aspergillosis & 1 \\
\hline Uterus & Retained products of conception & 2 \\
\hline
\end{tabular}

As regards etiology, pregnancy induced hypertension \& HELLP syndrome amounted to 7 cases, intrauterine fetal death 7 cases and postpartum hemorrhage 7 cases as the leading causes of RCN among obstetric causes, whereas septicemia 3 cases, hemolytic uremic syndrome 2 cases and poisoning 2 cases were the leading nonobstetric causes. [Tables $2 \mathrm{a} \& 2 \mathrm{~b}$ ]. Most of these cases presented with persistent oligo-anuria (80.8\%), fever 16 cases $(61.5 \%)$, bleeding from various sites 14 cases (53.9\%) and hypotension 12 cases (46.2\%). [Table 3]. Investigations revealed anemia, leukocytosis, thrombocytopenia, raised blood urea nitrogen (69.2\%), raised serum creatinine $(84.6 \%)$, altered coagulation profile $(53.9 \%)$ and raised bilirubin levels in 3 cases $(11.5 \%)$. [Table 4]. Ultrasound imaging was available only in 12 cases, depicting medico renal disease in 9, hypoperfusion in 3 cases, and a hypoechoic circumferential cortical band in 2 cases. 
Original Research Article

Grossly, both the kidneys were affected with swelling (57.7\%) while the single renal transplant case showed a mildly shrunken kidney and the rest of the cases, the kidneys were of normal sizes. Creamy-white areas of necrosis was seen in 17 (65.4\%) and unremarkable external appearance was seen in 9 cases (34.6\%) [Figure 1]. On microscopy, we found 14 autopsies (53.8\%) showing diffuse cortical necrosis while 12 autopsies $(46.2 \%)$ showed patchy cortical necrosis. The areas of renal cortical necrosis were characterized by coagulative necrosis of the glomeruli, tubules, interstitium and blood vessels. Structural details were preserved but cellular details like the nuclei were lost giving rise to a ghost - like appearance to the glomeruli. Inflammatory cell infiltration was also seen which varied from mild to dense in different cases. [Figure 2]. Viable renal parenchyma when present in the patchy RCN cases showed hemorrhages and congested vasculature. There were also thrombi seen in the arterioles and even the glomerular capillaries in some cases which were highlighted on the fibrin stain as deep orange color. [Figure 2].

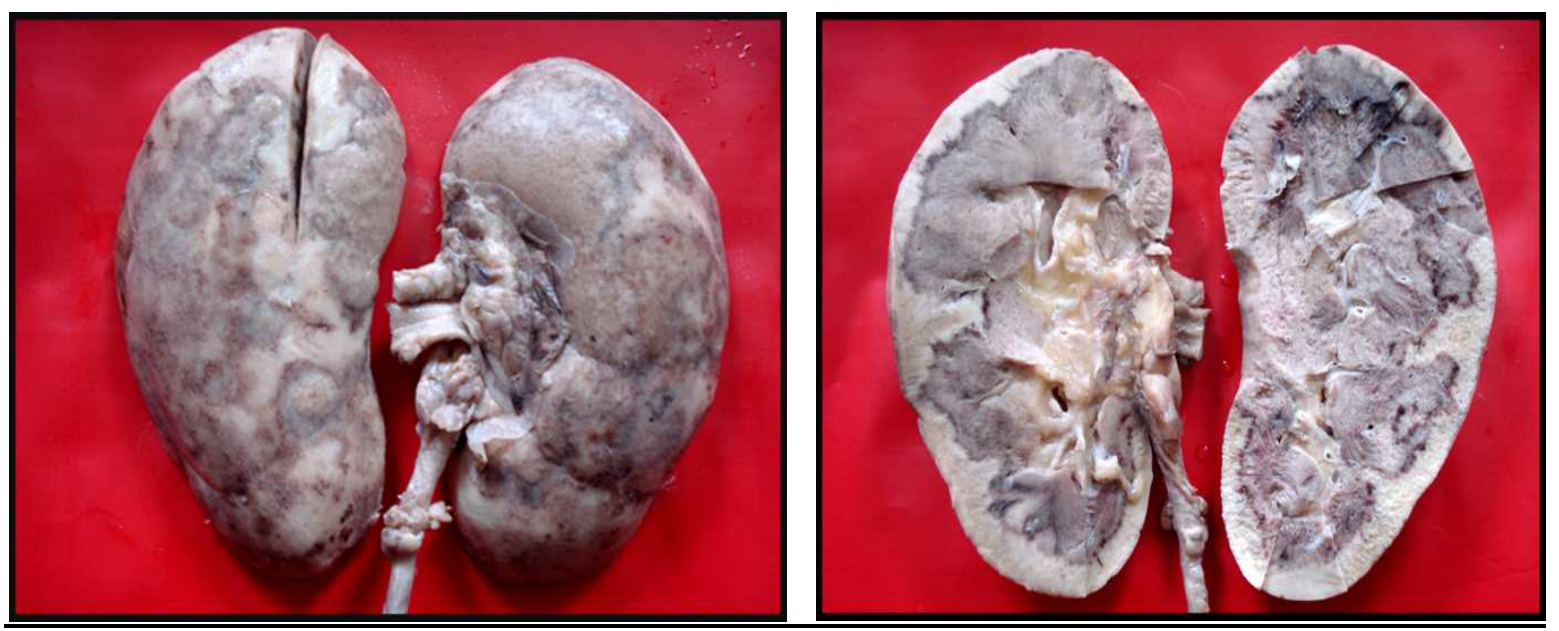

Fig-1: Gross morphology showing creamy-white, diffuse areas of cortical necrosis seen on the external and cut surface of the kidneys in a case of rejection of renal allograft

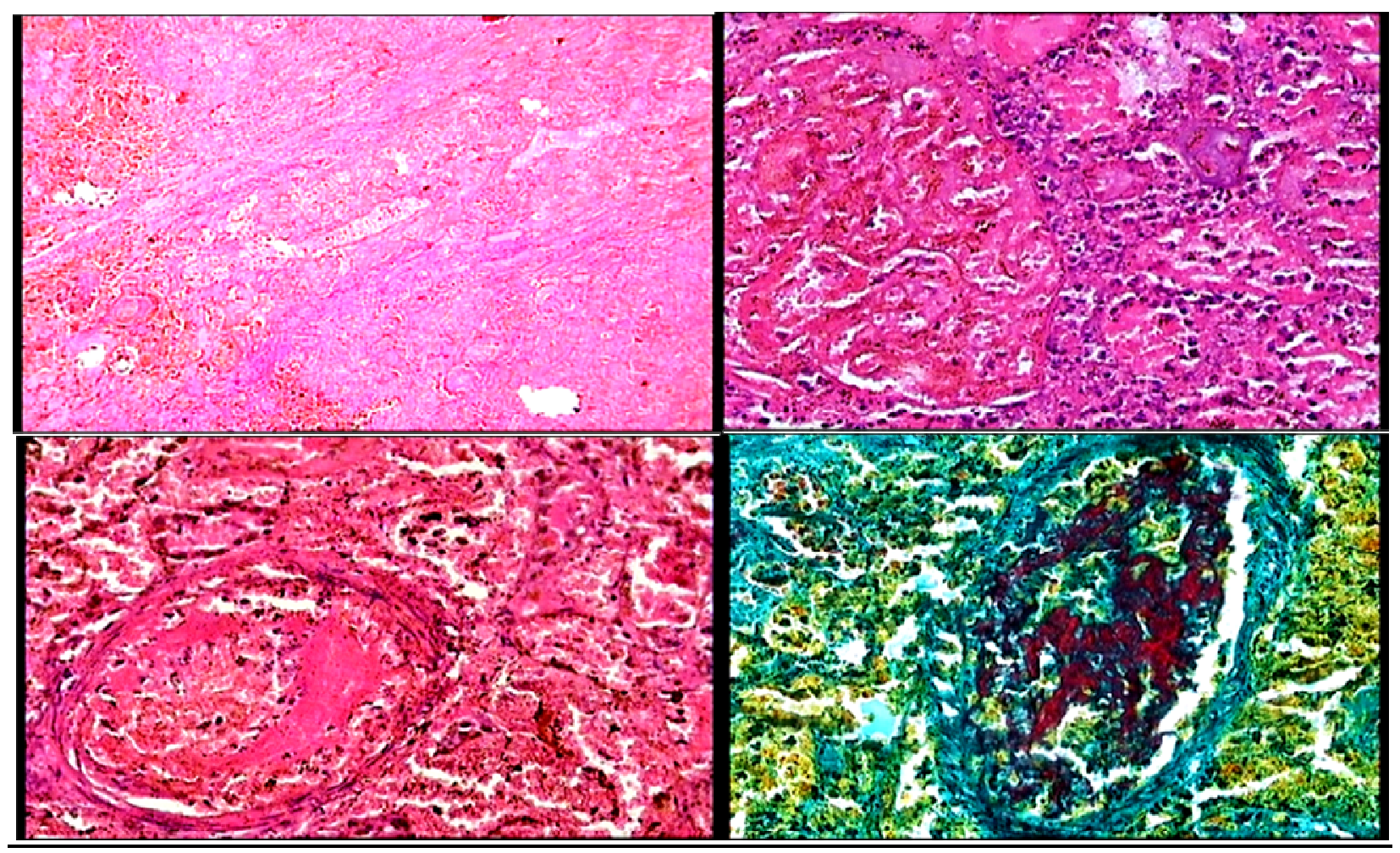

Fig-2: A - Extensive necrosis of the renal parenchyma. No viable renal parenchyma seen $(\mathrm{H} \& \mathbf{E x 1 0 0 )}$ 


\section{B - Coagulative necrosis of glomeruli and tubules surrounded by dense neutrophilic infiltrate. (H\&Ex400) \\ C - Thrombus occluding the vascular lumen (H\&Ex400) \\ D - Fibrin stain highlighting the thrombus in orange. (Fibrin $\mathbf{x 4 0 0 )}$}

The commonest findings on histopathology of other organs included intra-alveolar hemorrhage (26.9\%), bronchopneumonia (23.1\%), cerebral edema (20\%) and acute respiratory distress syndrome (15.4\%). [Table 5].

\section{Discussion}

This is a retrospective study of 26 complete autopsies of renal cortical necrosis. Almost all studies in literature on $\mathrm{RCN}$ are based on renal biopsy diagnosed RCN and autopsy studies are very few.

$\mathrm{RCN}$, though rare causes high morbidity and mortality and is fatal if untreated. In our study, we found the incidence of $\mathrm{RCN}$ to be $0.19 \%$ among 13,896 autopsies. The incidence of RCN reported in literature was found to be $0.2 \%$ in a study of 11,800 autopsies conducted by Kim Solez, three decades ago. Our incidence of RCN in AKI in general was $2.7 \%$.

Our incidence of $\mathrm{RCN}$ in $\mathrm{AKI}$ being $2.7 \%$ is significantly less than the $6-7 \%$ reported in literature in developing countries and could be due to the fact that this study was conducted at a tertiary care centre. RCN accounts for $6-7 \%$ of acute renal failure in developing countries, in contrast to $1.9-2 \%$ in developed countries. Jai Prakash et al, in a single centre experience have reported the incidence of $\mathrm{RCN}$ as $3.12 \%$ of all cases of Acute renal failure on renal biopsy [1]. Our incidence of RCN in maternal mortality autopsies was 2\% (17 cases) of 844 maternal mortality deaths during this period of study. We had $134(15.9 \%)$ maternal mortality deaths with AKI out of the 844 maternal mortality deaths.

The pathogenesis of RCN is complex and not well understood. Ischemic necrosis of the renal cortex results from decreased renal artery perfusion which is secondary to vasospasm, microvascular injury or disseminated intravascular coagulation [2]. The primary event in RCN is believed to be vasospam of small vessels, liberation of toxins and resultant endothelial injury. Two pathogenetic mechanisms have mainly been considered in the development of $\mathrm{RCN}$ [2]. One is renal hypoperfusion resulting from hypotension and the second is vascular endothelial injury which can work through a direct and indirect mechanism [3]. Hemolytic uremic syndrome, eccalmpsia, snake bite work through the direct mechanism while sepsis, intravascular hemolysis act by the indirect mechanism by release of endothelial- derived vasoconstrictor substances [2]. The exact pathogenesis is yet to be confirmed and the etiology may be multifactorial.

$\mathrm{RCN}$ has bimodal incidence, the first peak occurs during early infancy due to severe perinatal events [4] and the second peak in women of childbearing age due to obstetric complications. In our study, RCN did not afflict any particular age group and it was widely distributed from 4 days of life to 65 years. RCN equally affects both sexes in childhood whereas in adults it is more frequent in women due to obstetric complications. In our study, $80.8 \%$ of the cases were females with nearly all except one with obstetric causes.

The causes of RCN can be grouped into obstetric and non-obstetric. We had 17 obstetric cases (65.4\%) and 9 non-obstetric cases (34.6\%). We had 3 pediatric cases comprising of perinatal asphyxia, postoperative trachea-esophageal fistula repair and folliculitis leading to sepsis. RCN in childhood occurs secondary to perinatal complications, congenital heart disease, fetal-maternal transfusion, dehydration, severe hemolytic disease and sepsis and the commonest causes was Hemolytic uremic syndrome and sepsis in pediatric population [4,5].

Pregnancy induced hypertension with HELLP syndrome; intrauterine fetal death and post partum hemorrhage were the commonest obstetric causes of renal cortical necrosis encountered by us. Apart from it, other etiologies encountered were sepsis, abruptio placenta, spontaneous abortion and ruptured ectopic pregnancy. Obstetric catastrophies responsible for $\mathrm{RCN}$ in most reported series consists of abruptio placentae, septic abortion, eclamptic toxemia, PPH, IUFD, amniotic fluid embolism and puerperal sepsis [6,7,8,9]. In most 
literature series, abruptio placentae was the commonest etiology among obstetric causes of RCN. However, abruptio placentae was not the commonest cause noted in our study. We also found obstetric RCN to occur mainly in last trimester of pregnancy and in older multigravid women rather than primigravid women. The commonest non-obstetric cause in our study was septicemia followed by Hemolytic uremic syndrome and poisoning (1,4 dioxane and an unknown poison). $\mathrm{RCN}$ following exposure to 1,4 dioxane was one of our unusual cases of occupational hazards, where the patient was exposed to 1,4 dioxane following inhalation and skin contact after washing industrial tanks containing 1,4 dioxane. He developed anuria, diahorrea and respiratory distress within 6-8 hours of contact and succumbed within 4 days of acute respiratory distress syndrome, hepatic necrosis and acute renal failure. Another unusual case of RCN was seen following rejection of renal allograft, probably due to rejection. We had one postoperative case of RCN following a hysterectomy. The various non-obstetric causes of RCN reported in literature includes extensive burns, septicemia, snake bites, diabetic ketoacidosis, Hemolytic uremic syndrome, acute gastroenteritis, organophosphorus poisoning and surgical procedures $[8,10]$.

In our study, most patients presented with oligoanuria, fever, bleeding from various sites and hypotension. Jaundice was seen in 3 cases (11.5\%). Altered sensorium, unconsciousness and hepatic encephalopathy were seen in 5 cases $(19.2 \%)$. The clinical presentation in most studies included renal failure, hematuria, flank pain and on examination shows costo-vertebral tenderness with palpable tender kidneys [8]. Laboratory investigations often reveal anemia, leukocytosis, thrombocytopenia, raised blood urea nitrogen, serum creatinine, lactate dehydrogenase and altered coagulation profile as was seen in our study $[8,10,11]$. Every obstetric case which had the coagulation profile work-up done showed significant alterations of the coagulation profile.

Ultrasound imaging was available in 12 cases only and showed varying findings. 9 cases showed medico-renal disease, 3 showed hypoperfusion of the kidneys and 2 showed hypoechoic circumferential cortical band. Sonogram initially showed enlarged kidneys with reduced blood flow and shrunken cortical tissue with a characteristic hypoechoic circumferential band in the subcapsular area later on in RCN [12]. The radiological hallmark of thin cortical shell calcification on radiographs develops four to five weeks later.

On gross and microscopic examination, we found diffuse cortical necrosis $(53.8 \%)$ to be more common than the patchy of cortical necrosis (46.2\%). Jai Prakash et al have reported diffuse cortical necrosis as $71.9 \%$ and patchy cortical necrosis as $28 \%$ on renal biopsies [1]. Sahay et al have reported diffuse cortical necrosis in $28.6 \%$ and patchy cortical necrosis as $71.4 \%$ on renal biopsies [8]. Chugh et all have reported DCN in $62.8 \%$ and patchy in $37.2 \%$ on renal biopsies [13]. In a series of nine cases of RCN, Syed Sohail et al found patchy type $(77.8 \%)$ of $\mathrm{RCN}$ to be more common [14]. Patchy lesions of RCN are in general prevalent in most case series. It is easier to diagnose diffuse cortical necrosis on autopsy rather than on biopsy as the material is limited in biopsies.

The incidence of RCN in obstetric acute kidney injury in developing countries has been on a steady decrease since the last 2 to 3 decades. The incidence of RCN decreased from 6.7\% in 19841994 to $1.6 \%$ in 1995-2005 of the total ARF cases while RCN due to obstetric causes dropped from $4.7 \%$ in the $1990 \mathrm{~s}$ to $0.5 \%$ in the $2000 \mathrm{~s}$. [1]. Mortality rates at a single centre in the eastern India have shown a striking decline from $72 \%$ (1984-1994) to $19 \%(1995-2005)$ in the next decade [1]. In a study conducted by Prakash J, Pant $\mathrm{J}$ et al, the incidence of AKI has declined low enough to label it as a disappearing entity [15]. We have however encountered 17 autopsy cases of obstetric RCN of 13896 autopsies performed over a 12 year period at a tertiary care centre at a metropolis.

Mortality rate of $\mathrm{RCN}$ though rare is high and exceeds $50 \%$ in untreated cases. Early initiation of dialysis has significantly diminished this rate. 10 of our cases $(38.5 \%)$ were on dialysis during the antemortem period. In developing countries, overall decreasing rates are seen wherever health care facilities and dialysis have improved. The prognosis and survival of patients with cortical necrosis has improved markedly over years due to the prompt availability of dialysis, renal transplant facilities and overall better health care facilities. 
Therefore, swift diagnosis and treatment of the etiological factors of RCN and dialysis is of utmost importance, the failure of which is often fatal. Mortality rates due to renal cortical necrosis could well reflect the efficiency of health care services available.

\section{Conclusion}

$\mathrm{RCN}$, an uncommon but catastrophic entity, is known to produce high mortality if untreated. Our incidence of RCN was $0.19 \%$ at general autopsy, $2.7 \%$ in autopsies with AKI and $2 \%$ of maternal mortality deaths at a tertiary care centre. Our incidence of $\mathrm{RCN}$ in AKI being $2.7 \%$ is significantly less than the $6-7 \%$ reported in literature in developing countries. Obstetric complications were the leading cause with pregnancy induced hypertension and HELLP, postpartum hemorrhage and intrauterine fetal death. Diffuse pattern of cortical necrosis was more common than patchy cortical necrosis. The commonest findings, in order of frequency, on histopathology of other organs included intraalveolar hemorrhage, bronchopneumonia, cerebral edema and acute respiratory distress syndrome.

Funding: Nil, Conflict of interest: None Permission of IRB: Yes

\section{References}

1. Prakash J, Vohra R, Wani IA, Murthy AS, Srivastva PK, Tripathi K, Pandey LK, Usha, Raja $R$. Decreasing incidence of renal cortical necrosis in patients with acute renal failure in developing countries: a single-centre experience of 22 years from Eastern India. Nephrol Dial Transplant. 2007 Apr;22(4):1213-7. Epub 2007 Jan 31.

2. Waugh D, Manuel P. Serotonin-induced Acute Nephrosis and Renal Cortical Necrosis in Rats. A Morphologic Study with Pregnancy Correlations Am J Pathol. 1960 Apr;36(4): 431-455.

3. Yanagisawa M, Kurihara H, Kimura S, Tomobe Y, Kobayashi M, Mitsui Y, Yazaki Y, Goto K, Masaki T. A novel potent vasoconstrictor peptide produced by vascular endothelial cells. Nature. 1988 Mar 31;332(6163):411-5.

4. Lerner G, Kurnetz R, Bernstein J, Chang C et al. Renal cortical and renal medullary necrosis in the first 3 months of life. Pediatr Nephrol. 1992 Nov;6(6):516-8.

5. Prakash J, Sen D, Kumar NS, Kumar H, Tripathi LK, Saxena RK. Acute renal failure due to intrinsic renal diseases: review of 1122 cases. Ren Fail. 2003 Mar;25(2):225-33.

6. Prakash J, Kumar H, Sinha DK, Kedalaya PG, Pandey LK, Srivastava PK, Raja R, Usha. Acute renal failure in pregnancy in a developing country: twenty years of experience. Ren Fail. 2006;28(4):309-13.

7. Ali A, Ali M, Ali U. Obstetrical associated renal, cortical necrosis: though uncommon but not rare! J Ayub Med Coll Abbottabad. 2010;22(3)7476.

8. Sahay M; Swarnalata, Swain M, Padua M. Renal cortical necrosis in tropics. Saudi J Kidney Dis Transpl. 2013 Jul;24(4):725-30.

9. Naqvi R, Akhtar F, Ahmed E, Shaikh R, Ahmed Z, Naqvi A, Rizvi A. Acute renal failure of obstetrical origin during 1994 at one center. Ren Fail. 1996 Jul;18(4):681-3.

10. Prakash J, Pratap Singh V. Changing picture of renal cortical necrosis in acute kidney injury in developing country. World J Nephrol. 2015 Nov;4(5): 480-486. doi: 10.5527/wjn.v4.i5.480

11. Prakash J, Tripathi K, Pandey LK et al. Spectrum of renal cortical necrosis in acute renal failure in Eastern India. Postgrad Med J. 1995;71:208-10.

12. Jha V, Sakhuja V. Postpartum renal cortical necrosis. Images in nephrology. Nephrol Dial Transplant. 2005; 20(5):1010. doi:10.1093/ndt/gfh588

13. Chugh KS, Jha V, Sakhuja V, Joshi K. Acute renal cortical necrosis--a study of 113 patients. Ren Fail. 1994;16(1):37-47.

14. Ali SS, Rizvi SZ, Muzaffar S, Ahmad A, Ali A, Hassan SH. Renal cortical necrosis: a case series of nine patients \& review of literature. J Ayub Med Coll Abbottabad. 2003 Apr-Jun;15(2):41-4.

15. Prakash J, Pant P, Singh A, Sriniwas $S$ et al. Renal cortical necrosis is a disappearing entity in 
obstetric acute kidney injury in developing countries: our three decade of experience from
India. Ren Fail. 2015 Aug;37(7):1185-9. doi: 10.3109/0886022X.2015.1062340.

\section{How to cite this article?}

Fernandes Gwendolyn, Baskaran Sumitha, Fernandes Ellerhea. Renal cortical necrosis at autopsy: a 12 year experience at a tertiary care centre in Mumbai. Int J Med Res Rev 2017;5(06):626-634. doi:10.17511/ijmrr. 2017.i06.13. 